\title{
Self-cancellation of solar ephemeral regions observed by SDO
}

\author{
Shuhong Yang ${ }^{1}$, Jun Zhang ${ }^{1}$, Ting $\mathrm{Li}^{1}$ and Yang Liu ${ }^{2}$ \\ ${ }^{1}$ Key Laboratory of Solar Activity, National Astronomical Observatories, \\ Chinese Academy of Sciences, Beijing 100012, China \\ email: shuhongyang@nao.cas.cn \\ ${ }^{2}$ W.W. Hansen Experimental Physics Laboratory, Stanford University, \\ Stanford, CA 94305-4085, USA \\ email: yliu@sun.stanford.edu
}

\begin{abstract}
We study the ephemeral regions (ERs) in the quiet Sun observed by the Solar Dynamics Observatory, and find that they can be classified into two types: normal ERs (NERs) and self-cancelled ERs (SERs). We identify 2988 ERs among which there are 190 SERs, about $6.4 \%$ of the ERs. The total self-cancelled flux is $9.8 \%$ of the total ER flux. We suggest that the self-cancellation of SERs is caused by the submergence of magnetic loops connecting the dipolar patches, without magnetic energy release.
\end{abstract}

Keywords. Sun: activity, Sun: photosphere, Sun: surface magnetism

\section{Introduction}

Solar ephemeral regions (ERs) are small-scale dipolar magnetic structures with short lifetime and their properties have been investigated by many authors (Harvey \& Martin 1973; Schrijver et al. 1998; Hagenaar et al. 2003). Yang et al. (2009) studied magnetic field evolution in a coronal hole region, and reported that the dipolar patches of an ER separated first and then moved together and canceled with each other. Wang et al. (2012) studied the solar intranetwork magnetic fields and found an intranetwork dipolar flux emergence followed by cancellation of its two poles with opposite polarities. Based on statistical results, our present study is dedicated to know how often and to what extent the ERs in the quiet Sun perform the self-cancellation behavior, i.e., an ER canceling itself after emergence.

\section{Observations and Results}

We adopt the full-disk line-of-sight magnetograms obtained from 2010 June 11 12:00 UT to 2010 June 15 12:00 UT by the Helioseismic and Magnetic Imager (HMI; Scherrer et al. 2012) on board the Solar Dynamics Observatory (Pesnell et al. 2012). We differentially derotate all the magnetograms to a reference time, 2010 June 13 12:00 UT.

We track the ERs according to their evolution, and identify 2988 ERs during the four days. We find that these ERs can be classified into two types according to their performance: normal ERs (NERs) and self-canceled ERs (SERs). Each NER emerged and grew with separation of its dipolar patches, which canceled or coalesced with other magnetic flux eventually. Most of the ERs are NERs and the number of NERs is 2798 . Each SER emerged and its dipolar patches separated at first, but then a part of magnetic flux of the SER moved together and canceled gradually. Figure 1 shows the evolution of an SER as an example. There are only 190 SERs, about $6.4 \%$ of the ERs. 
14-Jun-2010 08:11:03 UT
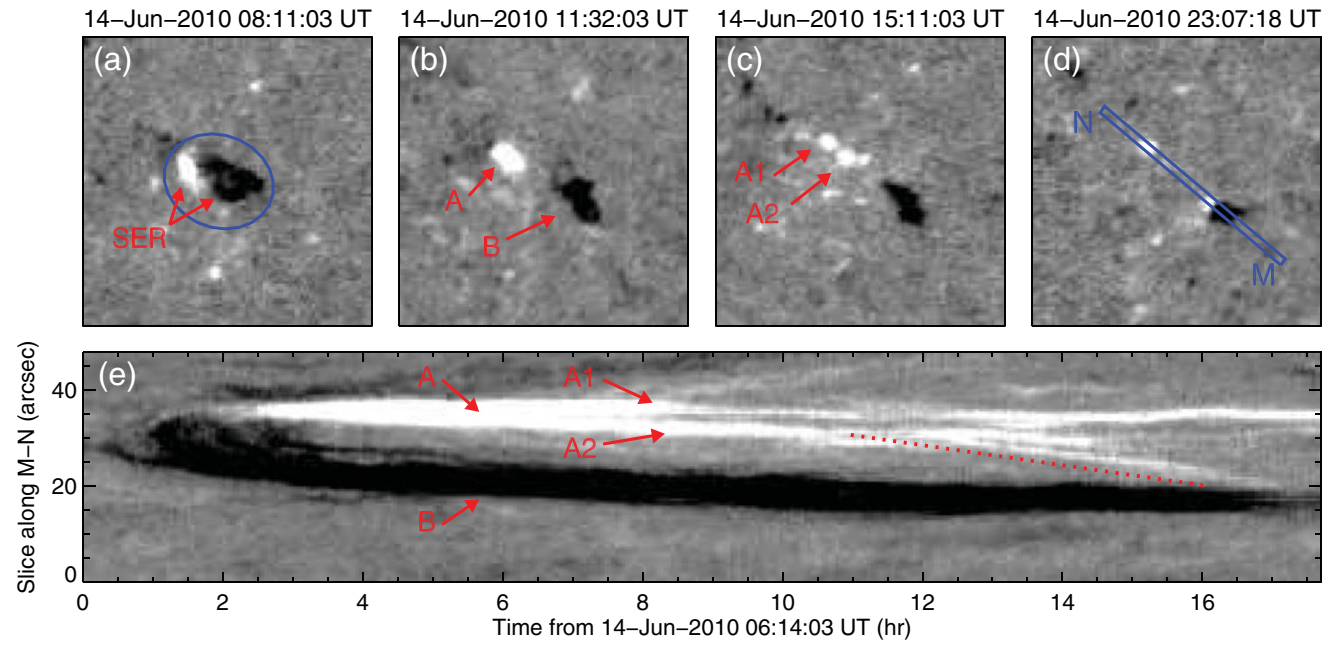

Figure 1. (a)-(d): Sequence of HMI magnetograms displaying the evolution of an SER. The field-of-view is $50 \times 50 \operatorname{arcsec}^{2}$. (e): space-time plot along slit $\mathrm{M}-\mathrm{N}$.

For SERs, only part of the magnetic flux is self-canceled. The mean value of selfcancellation fraction of SERs is $62.5 \%$, and the total self-canceled flux is $9.8 \%$ of the total ER flux. We also find that, the higher the ER magnetic flux is, (1) the easier the performance of ER self-cancellation is, (2) the smaller the self-cancellation fraction is, (3) the more the self-canceled flux is.

\section{Discussion}

Magnetic flux cancellation is a description of flux disappearance when two magnetic patches with opposite polarities encounter, so it is an observational phenomenon. In theory, the disappearance of magnetic flux can be resulted from the retraction of magnetic loops into the sub-photosphere, if the two poles are still connected by initial loops (Zwaan 1987). We suggest that the self-cancellation of SERs is caused by the submergence of magnetic loops connecting the dipolar patches. During this process, no magnetic reconnection occurs and no magnetic energy is released.

\section{Acknowledgements}

This work is supported by the National Natural Science Foundations of China (11203037, 11025315, 40890161, 10921303, 41074123 and 11003024), the CAS Project KJCX2-EW-T07, and the National Basic Research Program of China under grant $2011 \mathrm{CB} 811403$.

\section{References}

Hagenaar, H. J., Schrijver, C. J., \& Title, A. M. 2003, ApJ, 584, 1107

Harvey, K. L. \& Martin, S. F. 1973, Solar Phys., 32, 389

Pesnell, W. D., Thompson, B. J., \& Chamberlin, P. C. 2012, Solar Phys., 275, 3

Scherrer, P. H., Schou, J., Bush, R. I., et al. 2012, Solar Phys., 275, 207

Schrijver, C. J., Title, A. M., Harvey, K. L., et al. 1998, Nature, 394, 152

Wang, J. X., Zhou, G. P., Jin, C. L., \& Li, H. 2012, Solar Phys., 278, 299

Yang, S. H., Zhang, J., \& Borrero, J. M. 2009, ApJ, 703, 1012

Zwaan, C. 1987, ARAA, 25, 83 